\title{
The Harrison Model as a Tool to Study Phase Transitions in Magnetocaloric Materials
}

\author{
P. Gębara ${ }^{a}$, R. Gozdur ${ }^{b}$ And K. Chwastek ${ }^{c, *}$ \\ ${ }^{a}$ Institute of Physics, Faculty of Materials Processing and Production Technology, \\ Częstochowa University of Technology, Al. AK 19, 42-201 Częstochowa, Poland \\ ${ }^{b}$ Department of Semiconductor and Optoelectronic Devices, Łódź University of Technology, \\ Wólczańska 211/215, 90-924 Łódź, Poland \\ ${ }^{c}$ Faculty of Electrical Engineering, Częstochowa University of Technology, \\ al. Armii Krajowej 17, 42-201 Częstochowa, Poland \\ (Received October 24, 2018)
}

\begin{abstract}
Modeling of hysteresis loops close to the Curie point may shed some light on physics of magnetization processes in magnetocaloric materials. In the present paper the model advanced by Harrison is used to describe magnetization curves of $\mathrm{La}(\mathrm{Fe}, \mathrm{Co}, \mathrm{Si})_{13}$ alloy. Systematic study has revealed that values of some model parameters depend significantly on temperature. The trends of their variations are discussed in the paper.
\end{abstract}

DOI: 10.12693/APhysPolA.134.1217

PACS/topics: 75.30.Sg, 75.60.-d, 75.60.Ej

\section{Introduction}

The magnetocaloric effect observed in $\mathrm{La}(\mathrm{Fe}, \mathrm{Co}, \mathrm{Si})_{13}$ alloys in near-room temperature has attracted the attention of scientific community due to its potential applications in magnetic refrigeration as an alternative to conventional gas-vapor compression technique [1-6].

The study of phase transitions in magnetocaloric materials requires that new computational tools able to capture the effect of varying temperature on the shape of magnetization curve be developed [7-10]. In our opinion a convenient description for this purpose could be the Harrison model [11-13].

\section{The Harrison model - fundamentals}

The description is based on the assumption that field strength for any magnetization value might be decomposed into hysteretic and anhysteretic parts. The irreversible process leading to hysteresis occurs on the quantum scale. Cooperation between atomic moments leading to their head-to-tail alignment within a magnetic domain is expressed in this model with the coefficient $\beta$, whose value is related to the intrinsic coercivity of the material. Expression (1) relates magnetization $M$ to the applied field $H$ :

$$
M=M_{s} \tanh \left(\frac{\mu_{0} \beta \mu_{\mathrm{B}}}{k T}(H+\alpha M)\right),
$$

where $M_{s}[\mathrm{~A} / \mathrm{m}]$ is saturation magnetization, $\mu_{0}=4 \pi \times$ $10^{-7} \mathrm{H} / \mathrm{m}$ is free space permeability, $\mu_{\mathrm{B}}$ is the Bohr magneton, $\mu_{\mathrm{B}}=9.274 \times 10^{-24} \mathrm{~A} \mathrm{~m}^{2}, k=1.381 \times 10^{-23} \mathrm{~J} / \mathrm{K}$ is

\footnotetext{
* corresponding author; e-mail: krzysztof . chwastek@gmail.com
}

Boltzmann's constant, $\alpha$ is Weiss' coefficient accounting for the exchange field between atomic moments, whereas $T[\mathrm{~K}]$ is temperature.

It should be remarked that there exists a refined version of the description, which avails of the Brillouin function instead of hyperbolic tangent on the right hand side of the expression (1) [12]. The use of the Brillouin function in magnetism is well justified, but in the present paper we retain the original form in order to keep the number of model parameters as low as possible (by not introducing yet another parameter, namely the angular momentum quantum number $J$ ). In this way we simplify the computations and avoid the necessity to invert the Brillouin function numerically. Its two limiting cases are the Langevin expression $L(x)=\operatorname{coth}(x)-1 / x$, which corresponds to $J \rightarrow \infty$ and hyperbolic tangent, which describes the case $J=1 / 2[4,5]$.

Numerical solution of Eq. (1) leads to an S-shaped curve on the $M-H$ plane, that passes through the second and the fourth quadrants. In these parts of the $M-H$ plane there are two bifurcation points, at which the derivative $\partial H / \partial M$ becomes zero. The middle part of the magnetization curve (with negative susceptibility) is normally not observable. However at this point the results of experimental research carried out by Helmiss and Storm [16] and by Grosse-Nobis [17] on single iron crystals with one movable Bloch wall should be recalled. These authors reported on constricted hysteresis loops, whose middle parts are similar to that obtained from the solution of Eq. (1).

There exists an analogy between the qualitative behaviour of the model given with expression (1) and of the double-well bistable system, whose free energy under non-zero input is given with the equation [18]:

$$
g_{L}(x, h)=x^{4}-2 a x^{2}-h x .
$$


In the latter system energy minima for zero field are located at $x= \pm \sqrt{a}$. Under the generic field $h$ the metastable states are identified from the condition $\partial g_{L} / \partial x=0$, with $\partial^{2} g_{L} / \partial x^{2}>0$, identifying local $g_{L}$ minima. Figure 1 depicts schematically the energy profiles of the bistable system for different values of external field at fixed temperature.

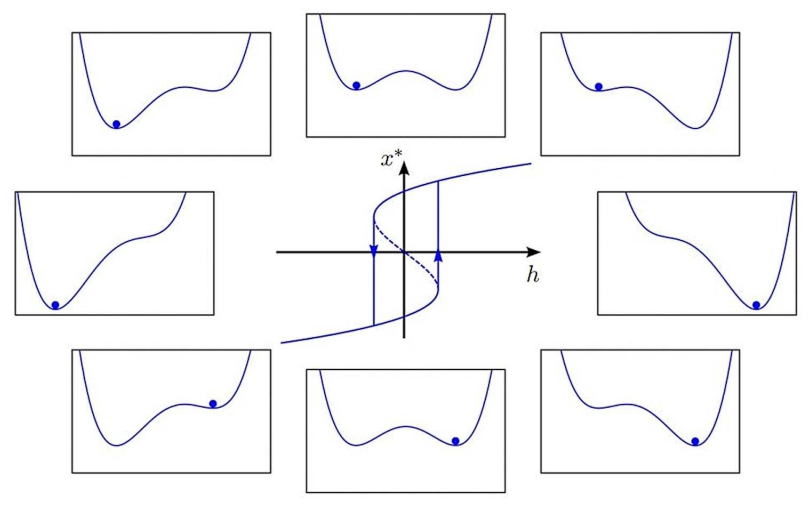

Fig. 1. Hysteresis loop of a bistable system (the unstable part marked with dashed line) and a sequence of energy profiles for different values of external field.

Equation (1) may be expressed in dimensionless form as [11]:

$$
y=\tanh \left(\frac{x+y}{t}\right),
$$

which allows one to express $x$ as function of $y$ :

$$
x=\operatorname{tarctanh}(y)-y \text {. }
$$

In the relationship above $y$ stands for reduced magnetization, $x$ is external field normalized with respect to $\alpha M_{s}$, whereas $t=T / T_{\mathrm{C}}$ is temperature in dimensionless units (referred to the Curie point).

The bifurcation points occur for $\partial x / \partial y=0$, thus

$$
\frac{t}{1-y_{c}^{2}}-1=0 \text {. }
$$

After solving the above given equation for $y_{c}$, one obtains $y_{c}= \pm \sqrt{1-t}$. Substitution of this value into expression (4) allows one to determine the $x_{c}$ coordinates.

According to Harrison, reversible magnetization process occurs on the mesoscopic (domain) level. The anhysteretic curve given with the inverse Langevin function accounts for these effects. In the paper we choose the Cohen approximation $[14,19]$ for its description

$$
H_{\mathrm{anh}}=\gamma m \frac{3-m^{2}}{1-m^{2}},
$$

where $\gamma$ is the pinning coefficient in Harrison's notation and $m=M / M_{s}$ is the reduced magnetization.

At this point it is expedient to take a closer look at formal and conceptual differences between the Harrison description and the Jiles-Atherton (JA) model [20]. In the JA approach domain wall translation through pinning centres (structural inhomogeneities) is responsible for irreversible magnetization processes (occurrence of hysteresis). In the Harrison model domain wall pinning does not appear to be the primary cause of hysteresis in ferromagnetics even for soft materials with sigmoidal loops (cf. [11], p. 958).

In the JA formalism the anhysteretic curve is given with the Langevin function, whose argument is the socalled effective field $H_{\text {eff: }}$

$$
M_{\mathrm{anh}}=M_{s}\left(\operatorname{coth} H_{\mathrm{eff}} / a-a / H_{\mathrm{eff}}\right),
$$

where $a$ is a temperature-dependent parameter. Usually the effective field is defined with the expression $H_{\text {eff }}=H+\alpha M$ ( $\alpha$ is the Weiss mean field parameter), but there are some papers, which redefine it to $H_{\text {eff }}=H+\alpha M_{\text {anh }}[21-23]$. In the first case the "anhysteretic" curve exhibits in fact hysteresis, in the latter one - there exists an implicit dependence between applied field $H$ and $M_{\text {anh }}$, whose graphical representation on the $M-H$ plane (passing through the second and the fourth quadrant) may resemble the S-shaped curve obtained from solution of Eq. (1) [24]. The model developers themselves depict in their landmark paper [20] (p. 52) an exemplary hysteresis loop resulting from solution of Eq. (7). This effect is explained by the assumption of excessive $\alpha$ value. In the JA theory it is assumed that total magnetization is obtained from summing irreversible and reversible contributions, the role of a weight is played by the model parameter $c$. This assumption has been criticized [24].

At this point it should be remarked that there exists a modification of the JA description, which relies rather on summation of field strengths than magnetization terms $[25,26]$. This version has a number of distinctive features: hysteresis loop branches are obtained by the introduction of an offset along the $H$ axis and not along the $M$-axis like in the original description [27], which results in a qualitatively different model behaviour for sudden field reversals [28]. The irreversible and reversible processes are described with decoupled sets of equations, like in the Harrison proposal (in the original JA approach they are coupled with the effective field). We have reported on encouraging results of modeling temperature-dependent hysteresis curves for $\mathrm{La}(\mathrm{Fe}, \mathrm{Co}, \mathrm{Si})_{13}$ with the modified JA approach in the paper [29].

\section{Measurements}

Magnetic properties of $\mathrm{La}(\mathrm{Fe}, \mathrm{Co}, \mathrm{Si})_{13}$ samples made of sintered fine-grained powders (particle size $<5 \mu \mathrm{m}$ ) were determined using a bridge method [30]. Quasi-static excitation conditions were checked using the classification criterion discussed in detail in Ref. [31]. More details on the measurement setup developed by one of the authors may be found in Ref. [4].

\section{Modeling}

The anhysteretic curve was identified from measured hysteresis loops at different temperatures as the middle curve [32]. This allowed us to determine the values of 
pinning coefficient $\gamma$ for the ferro- and paramagnetic regions. Theoretically the $\gamma$ value should remain constant; however we have noticed its sudden increase after crossing the Curie point ( $300 \mathrm{~K}$ for the examined sample). The trend is depicted in Fig. 2, fitted with the exponential growth function. It is assumed that this parameter might be a measure of disorder in the material.

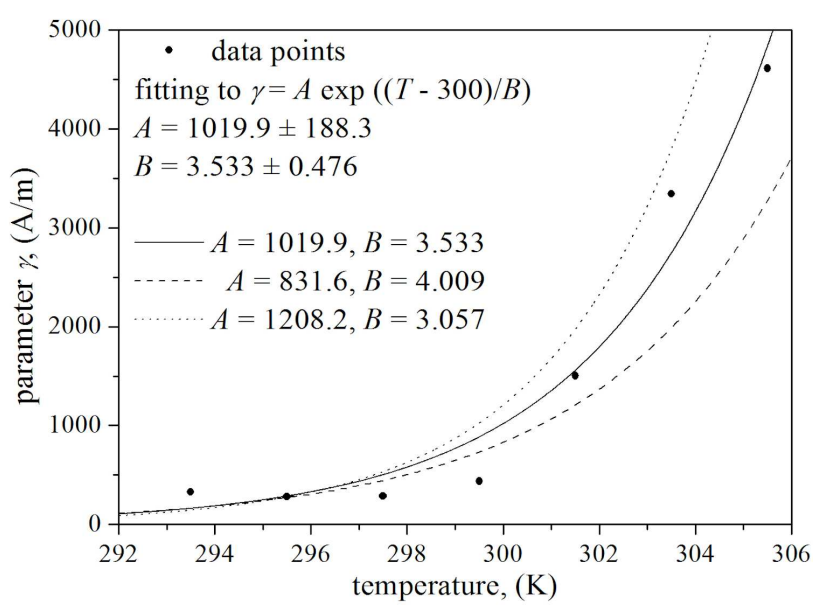

Fig. 2. The dependence $\gamma$ vs. temperature.

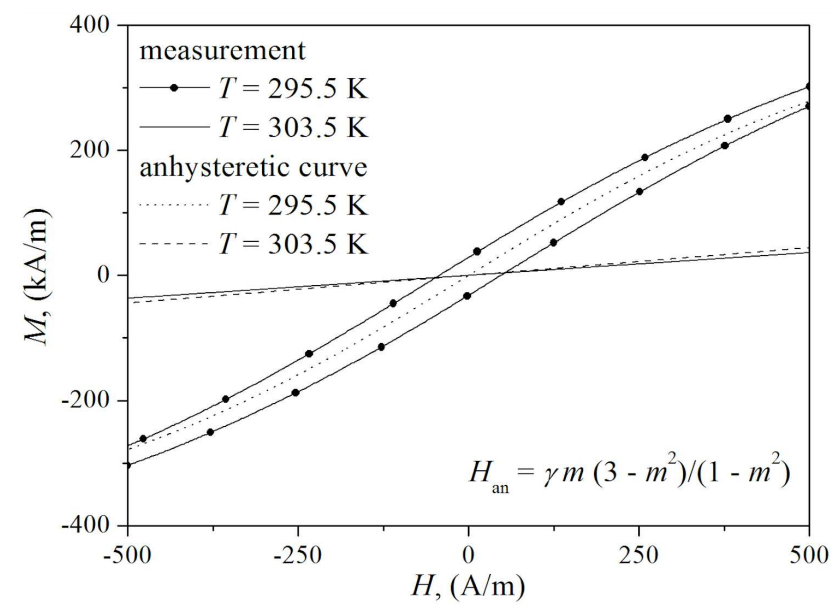

Fig. 3. The anhysteretic curves for two chosen temperatures.

In Fig. 3 experimental data points and anhysteretic curves modelled with the Cohen relationship, Eq. (6), are shown for two temperatures. The values of $\gamma$ were determined from the relationship shown in Fig. 2, the values $A=1019.9 \mathrm{~A} / \mathrm{m}$ and $B=3.533 \mathrm{~K}$ were chosen.

Experimental dependences $H_{c}=H_{c}(T)$ and $M_{s}=$ $M_{s}(T)$ were used for determination of the optimal values for the parameters $\beta$ and $\alpha$. Under assumption that field strengths at coercive and at bifurcation points are equal, it is possible to determine the $\alpha$ and $\beta$ values from experimental dependences $H_{c}=H_{c}(T)$ and $M_{s}=M_{s}(T)$. The experimental data points may be inserted directly into Eq. (1) and the solution may be found e.g. using optimization routines embedded in a spreadsheet.
It was found that $\beta$ decreased upon temperature increase. The depedence $\alpha$ vs. $T$ revealed an increasing trend, like in the case of parameter $\gamma$. Figure 4 depicts the modelled values (points) and their trends (lines), whereas Fig. 5 presents measured and modeled hysteresis curves for $T=293.5 \mathrm{~K}$.

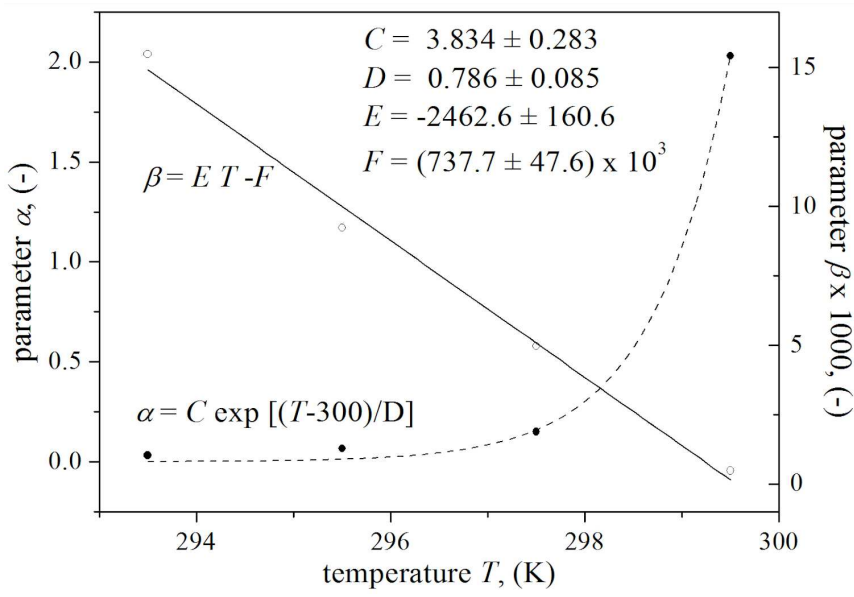

Fig. 4. The dependences $\alpha$ and $\beta$ vs. temperature.
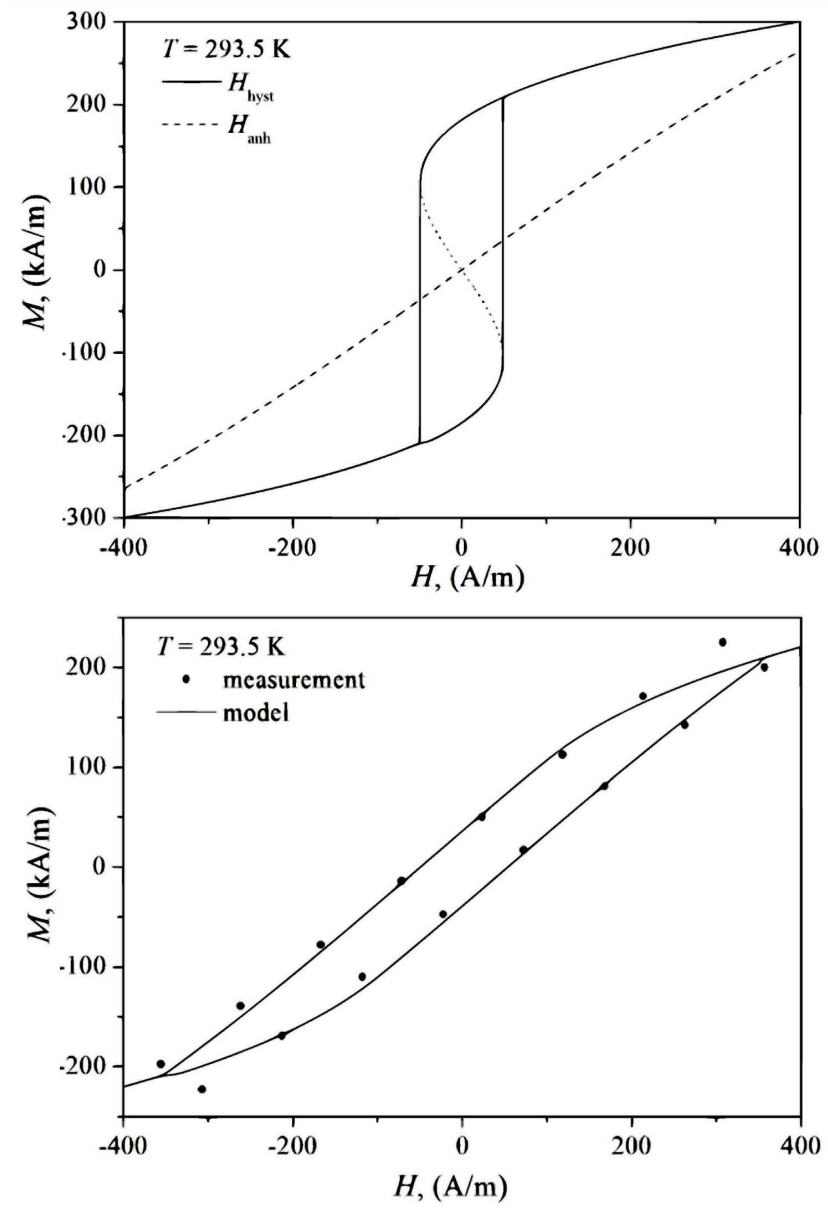

Fig. 5. Exemplary hysteresis curves for an arbitrary temperature. 


\section{Conclusions}

In the paper we have applied the Harrison model for the description of magnetization curves in $\mathrm{La}(\mathrm{Fe}, \mathrm{Co}, \mathrm{Si})_{13}$ alloy for temperatures close to the Curie point. The formalism relies on separation of reversible and irreversible contributions, which act on different spatial scales. It was found that some model parameters $\left(\alpha, \beta, \gamma\right.$ and $\left.M_{s}\right)$ are dependent on temperature. The values of $\alpha$ and $\gamma$ increase abruptly; the observed trends may be described with the exponential growth function. The parameter $\beta$ decreases linearly upon temperature increase.

\section{Acknowledgments}

This research was partly supported by the grants No. $6370 / \mathrm{B} / \mathrm{T} 02 / 2011 / 40$ and $4889 / \mathrm{B} / \mathrm{T} 02 / 2011 / 40$, from the National Science Centre of Poland.

\section{References}

[1] J. Liu, J.D. Moore, K.P. Skokov, M. Krautz, K. Löwe, A. Barcza, M. Katter, O. Gutfleisch, Scr. Mater. 67, 584 (2012).

[2] V. Lollobrigida, V. Basso, F. Borgatti, P. Torelli, M. Kuepferling, M. Coïsson, E.S. Olivetti, F. Celegado, L. Tortora, G. Stefani, G. Panaccione, F. Offi, J. Appl. Phys. 115, 203901 (2014).

[3] P. Gębara, P. Pawlik, I. Škorvánek, J. Marcin, K. Pawlik, A. Przybył, J.J. Wysłocki, M. Szwaja, K. Filipecka, Acta Phys. Pol. A 126, 166 (2014).

[4] R. Gozdur, M. Lebioda, Ł. Bernacki, Acta Phys. Pol. A 128, 98 (2015).

[5] M. Balli, S. Jandl, P. Fournier, A. Kedous-Lebouc, Appl. Phys. Rev. 4, 021305 (2017).

[6] P. Gębara, J. Kovač, Mater. Des. 129, 111 (2017).

[7] D.P. Landau, Theory of Magnetic Phase Transitions in: Handbook of Magnetism and Advanced Magnetic Materials, Eds. H. Kronmüller, S. Parkin, M. Fähnle, S. Maekawa, I. Zutic, Vol. 1, Fundamentals and Theory, Wiley, New York 2007.

[8] V. Franco, J.S. Blázquez, B. Ingale, A. Conde, Annu. Rev. Mater. Res. 42, 305 (2012).

[9] A. Berti, C. Giorgi, E. Vuk, Appl. Math. Model. 39, 820 (2015).
[10] O. Gutfleisch, T. Gottschall, M. Fries, D. Benke, I. Radulov, K.P. Skokov, H. Wende, M. Gruner, M. Acet, P. Entel, M. Farle, Philos. Trans. A Math. Phys. Eng. Sci. 374, 20150308 (2016).

[11] R.G. Harrison, IEEE Trans. Magn. 39, 950 (2003).

[12] R.G. Harrison, J. Appl. Phys. 115, 033901 (2014).

[13] K. Chwastek, J. Szczygłowski, W. Wilczyński, Acta Phys. Pol. A 121, 941 (2012).

[14] A.S. Arrott, J. Appl. Phys. 103, 07 C715 (2008).

[15] K. Chwastek, J. Szczygłowski, Arch. Electr. Eng. 60, 49 (2011).

[16] G. Helmiss, L. Storm, IEEE Trans. Magn. 10, 36 (1974).

[17] W. Grosse-Nobis, J. Magn. Magn. Mater. 4, 247 (1977).

[18] G. Bertotti, Hysteresis in Magnetism, Academic Press, San Diego 1998.

[19] A. Cohen, Rheol. Acta, 30, 270 (1991).

[20] D.C. Jiles, D.L. Atherton, J. Magn. Magn. Mater. 61, 48 (1986)

[21] D.C. Jiles, J. Appl. Phys. 76, 5849 (1994).

[22] J. Szczygłowski, J. Magn. Magn. Mater. 223, 97 (2001).

[23] Liu Qingyou, Luo Xu, Zhu Haiyan, Liu Jianxun, Han Yiwei, Chin. Phys. B 26, 077502 (2017).

[24] S.E. Zirka, Yu.I. Moroz, R.G. Harrison, K. Chwastek, J. Appl. Phys. 112, 043916 (2012).

[25] P.I. Koltermann, L.A. Righi, J.P.A. Bastos, R. Carlson, N. Sadowski, N.J. Batistela, Physica B, 275, 233 (2000).

[26] L.A. Righi, N. Sadowski, R. Carlson, J.P.A. Bastos, N.J. Batistela, IEEE Trans. Magn. 37, 3353 (2001).

[27] R. Jastrzębski, K. Chwastek, I. Biondić, K. Miličević, Acta Phys. Pol. A 131, 1228 (2017).

[28] R. Jastrzębski, K. Chwastek, ITM Web Conf. 15 03003 (2017)

[29] R. Gozdur, P. Gębara, K. Chwastek, Open Phys. 16 266 (2018).

[30] A. Majocha, R. Gozdur, Przegl. Elektrotechn. 4, 79 (2010).

[31] R. Gozdur, A. Majocha, Przegl. Elektrotechn. 1, 134 (2007).

[32] J. Krah, A.J. Bergqvist, Physica B 343, 35 (2004). 